\title{
PSA: A Novel Optimization Algorithm Based on Survival Rules of Porcellio Scaber
}

\author{
Yinyan Zhang \\ College of Cyber Security \\ Jinan University \\ Guangzhou, China \\ Email: yyzhang@jnu.edu.cn
}

\author{
Pei Zhang \\ College of Cyber Security \\ Jinan University \\ Guangzhou, China \\ Email:1207382268@qq.com
}

\author{
Shuai Li \\ College of Engineering \\ Swansea University \\ Swansea SA1 7EN, U.K. \\ Email: shuaili@ieee.org
}

\begin{abstract}
Bio-inspired algorithms such as neural network algorithms and genetic algorithms have received a significant amount of attention in both academic and engineering societies. In this paper, based on the observation of two major survival rules of a species of woodlice, i.e., porcellio scaber, we present an algorithm called the porcellio scaber algorithm (PSA) for solving general unconstrained optimization problems, including differentiable and non-differential ones as well as the case with local optima. Numerical results based on benchmark problems are presented to validate the efficacy of PSA.

Index Terms-Bio-inspired algorithm, Optimization, Porcellio scaber, Benchmark problem.
\end{abstract}

\section{INTRODUCTION}

In recent decades, bio-inspired algorithms, such as neural networks, genetic algorithms [1], are widely investigated and applied in the fields of pattern recognition [2], robotics [3], control [4], task scheduling [5], and optimization [6]. Compared with traditional methods, bio-inspired algorithms are more efficient in handling complicated optimization problems, such as the NP-hard traveling salesman problem. Kennedy and Eberhart [7] proposed particle swarm optimization, which is one of the well known bio-inspired algorithms. It is based on the swarm behavior of animals, such as fish and bird schooling. Inspired by how ants find a shortest path, Dorigo and Gambardella [8] proposed an ant colony system algorithm for solving the traveling salesman problem. Based on some idealized behaviors of the flashing characteristics of fireflies, Yang et al. [9] proposed a firefly algorithm for solving non-convex economic dispatch problems with valve loading effect. Inspired by the competitive and selfinterested behaviors of political party individuals, Huan et al. [10] proposed a so-called ideology algorithm for solving unconstrained optimization problems. The performance of these algorithms indicates that bio-inspired algorithms can be efficient and promising.

In this paper, based on the observation of two survival rules of a species of woodlice, i.e., porcellio scaber (PS) [11], [13], [14], we present a novel algorithm called the porcellio scaber algorithm (PSA) for solving general unconstrained optimization problems including differentiable and non-differentiable ones as well as the case with local optima. The performance of the proposed algorithm is evaluated via benchmark problems.

\section{PORCELlio SCABER-INSPIRED ALGORITHM DESIGN}

Porcellio scaber, as shown in Fig. 1 is a species of woodlice. They prefer to live in moist, dark, and cool places and are known to live in groups [11]. Due to the outstanding behavioral advantage of porcellio scaber, some of its varieties even can survive in extremely harsh environments, e.g., deserts around North Africa and the Middle East, where the searching of moist, dark, and cool places is usually difficult [13]. To avoid desiccation and a rapid drop in temperature, both of which could cause significant mortality, they shelter. In addition to their eyes, porcellio scaber have many sensory receptors on their body, making it possible for them to detect chemical, mechanical, and hygrometric conditions about the environment [14]. When the environment conditions are not favourable, porcellio scaber keep moving until morality or reach places with good conditions. Besides, for worse environment conditions, they move more quickly. On the other hand, when the environment condition is optimal, i.e., sufficiently good, they stay there.

For the sake of illustration, let the position of the mass center of each porcellio scaber at the $k$ th $(k=0,1,2, \cdots)$ time instant be denoted by a vector $\mathbf{x}_{i}^{k}$ and let the environmental condition at a position $\mathrm{x}$ by described by a fitness function $f(\mathbf{x})$, where a minimum value of $f(\mathbf{x})$ corresponds to the optimal environmental condition for porcellio scaber. We consider the case that there is a group of totally $N$ porcellio scaber, i.e., $i \in\{1,2, \cdots, N\}$.

Many studies reveal that porcellio scaber have two behaviors, which are viewed as their survival rules. The two behaviors are depicted in Fig. 2. One is called aggregation and the other is called the propensity to explore novel environments [11], [14], [15]. Note that they aggregate at the places with good environment conditions [15]. To model 


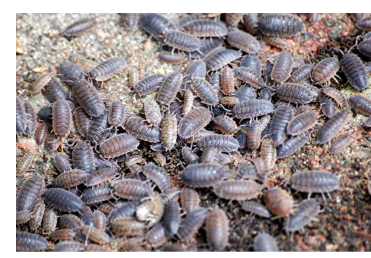

Fig. 1. Porcellio scaber by Peter Rühr, used under the Creative Commons Attribution 3.0 Unported license [12].

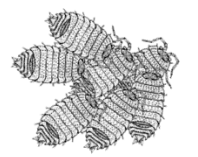

(a)

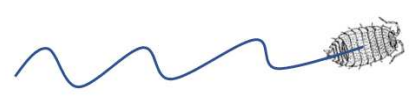

(b)

Fig. 2. Two behaviors of porcellio scaber. (a) Group behavior: aggregation. (b) Individual behavior: propensity to explore novel environments.

the aggregation behavior, we can describe the movement of porcellio scaber as follows:

$$
\mathbf{x}_{i}^{k+1}=\mathbf{x}_{i}^{k}-\left(\mathbf{x}_{i}^{k}-\arg \min _{\mathbf{x}_{j}^{k}}\left\{f\left(\mathbf{x}_{j}^{k}\right)\right\}\right)
$$

which can be rewritten as

$$
\mathbf{x}_{i}^{k+1}=\arg \min _{\mathbf{x}_{j}^{k}}\left\{f\left(\mathbf{x}_{j}^{k}\right)\right\} .
$$

Evidently, according to (1), each porcellio scaber will finally stay at the same position with the best environmental condition among all their initial positions $\mathbf{x}_{i}^{0}$. With only the aggregation behavior, porcellio scaber cannot live in case that they are initially placed at bad environment. Studies show that, each porcellio scaber has the propensity to explore novel environments [14]. Thus, the actual movement of each porcellio scaber is a weighted result of aggregation and the propensity to explore novel environments. By considering this behavior, we modify the model (1) and present the following PSA update rule to solve the problem :

$$
\mathbf{x}_{i}^{k+1}=\mathbf{x}_{i}^{k}-(1-\lambda)\left(\mathbf{x}_{i}^{k}-\arg \min _{\mathbf{x}_{j}^{k}}\left\{f\left(\mathbf{x}_{j}^{k}\right)\right\}\right)-\lambda p \tau
$$

where $p$ is a function to map the fitness of a porcellio scaber to an action strength. A simple choice is $p=f\left(\mathbf{x}_{i}^{k}+\tau\right)$. As verified in our extensive experiments, the following normalized fitness can achieve better performance:

$$
p=\frac{f\left(\mathbf{x}_{i}^{k}+\tau\right)-\min \left\{f\left(\mathbf{x}_{i}^{k}+\tau\right)\right\}}{\max \left\{f\left(\mathbf{x}_{i}^{k}+\tau\right)\right\}-\min \left\{f\left(\mathbf{x}_{i}^{k}+\tau\right)\right\}}
$$

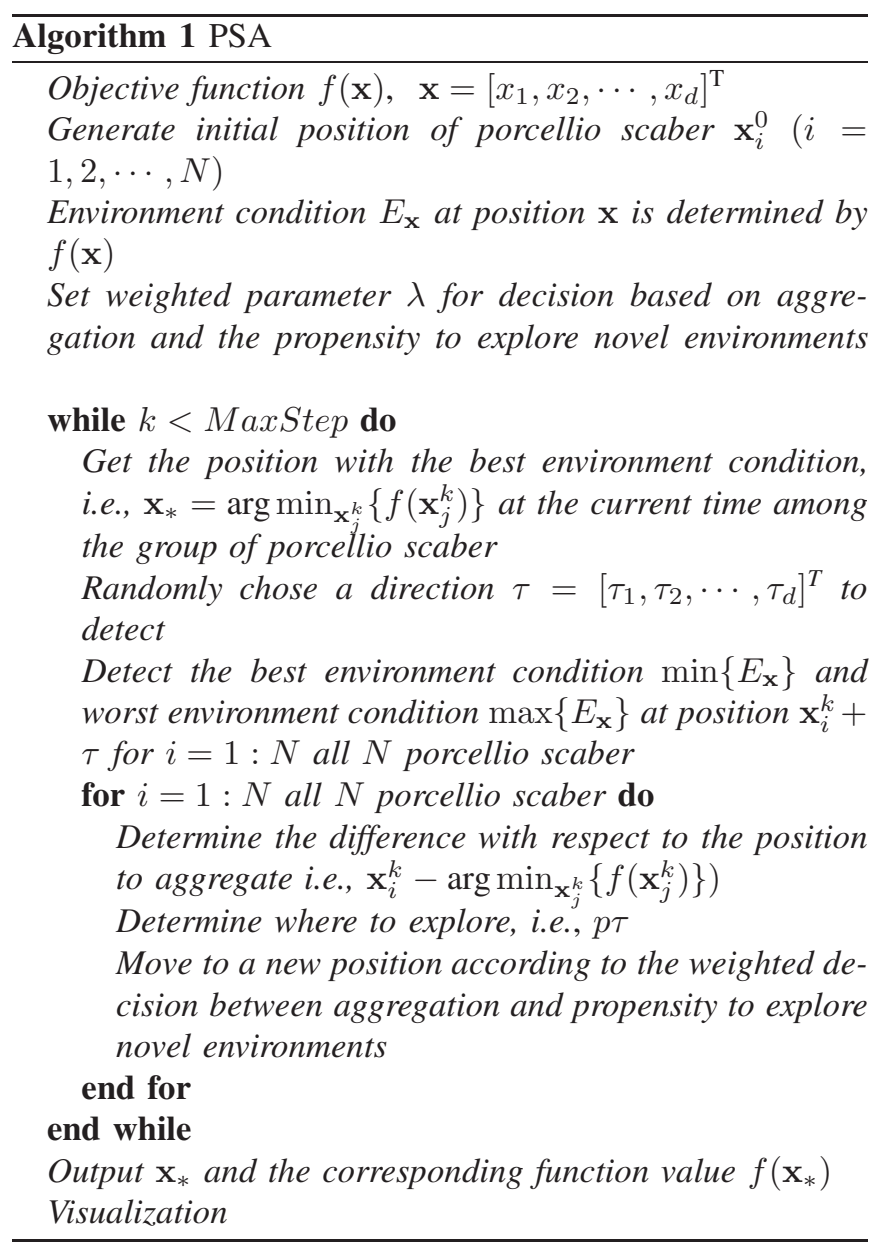

In the model (2), the term $\lambda \in(0,1)$ accounts for the weight between aggregation and the propensity to explore novel environments. The value of $\lambda$ can be different for each different porcellio scaber. The term $p \tau$ corresponds to the propensity to explore novel environments, where $\tau$ is a random vector that has the same dimension with that of $\mathbf{x}_{i}^{k}$. Specifically, $p \tau$ means that each porcellio scaber randomly chooses a direction with respect to their positions of mass centers to detect the environment condition of surroundings. In addition, the $p$ term means movement speed requirement which indicates that the exploration for novel environments is also a result of group negotiation.

The optimization problem considered in this paper is depicted as follows:

$$
\min _{\mathbf{x}} f(\mathbf{x})
$$

where function $f$ is lower bounded but can be nondifferentiable or non-convex and can have multiple local minima. By the above observations about the behaviors of porcellio scaber, the PSA for solving optimization problem (3) is designed and depicted in Algorithm 1. The main idea is to view the function to be minimized as an evaluation of 


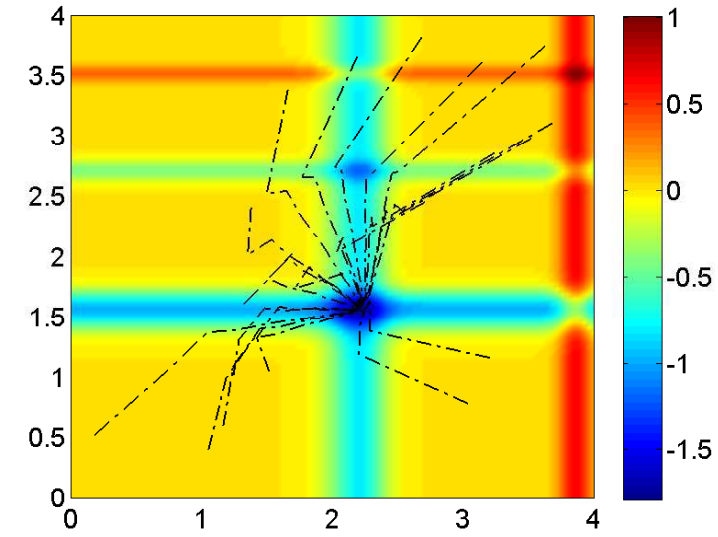

Fig. 3. Simulation result of the proposed PSA for finding the minimizer of the Michalewicz function (4) by using 20 porcellio scaber starting from randomly generated initial positions and by taking 40 steps of movement.

the environmental condition from the perspective of porcellio scaber, and view the decision variable as the position vector of a porcellio scaber. As the movement result of porcellio scaber is to stay at a place with the best environment condition, the algorithm design based on the observation of the movement behaviors of porcellio scaber is expected to be successful.

About the problem considered in this paper, we have the following remark.

Remark 1: The unconstrained optimization problem considered in this paper is general as it can be non-differentiable and non-convex. It should be noted that the differentiability of the objective function and the convexity of the problem are often assumed to be satisfied for existing optimization methods, such as [16], [17].

\section{BENCHMARK VALIDATION}

To test the efficacy of the proposed PSA, some benchmark problems are used.

\section{A. Example 1}

We consider the Michalewicz function [18]:

$$
f(\mathbf{x})=-\sum_{i=1}^{d} \sin \left(x_{i}\right)\left[\sin \left(\frac{i x_{i}^{2}}{\pi}\right)\right]^{2 m}
$$

where $m=10$ and $d=1,2, \cdots$. When $d=2$, the global minimum of the Michalewicz function is $f_{*} \approx-1.801$ which occurs at $\mathbf{x}_{*} \approx(2.2032,1.5705)$. Under the setting that each element of $\tau$ is a zero-mean random number with the standard deviation being 0.001 , with $\lambda=0.8,20$ porcellio scaber, and 40 steps, i.e., $N=20$ and MaxStep $=40$, the best numerical experiment result is visualized in Fig. 3. As seen from this figure, although there are some local minimums, all the porcellio scaber aggregate at about the optimal one.

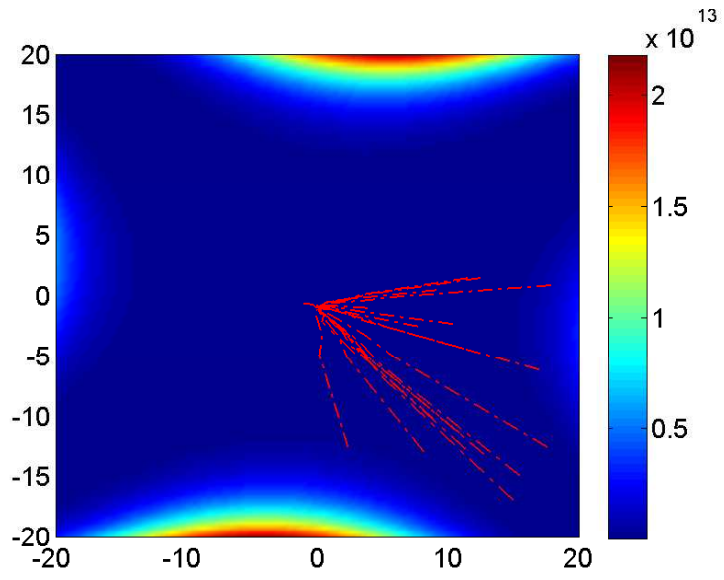

Fig. 4. Visualization of the numerical result of the proposed PSA for solving the Goldstein and Price problem [5 by using 20 porcellio scaber starting from randomly generated initial positions and by taking 40 steps of movement.

Numerically, the solution obtained in the numerical experiment is $\mathbf{x}_{*}=[2.202847772916551,1.570778088262819]^{\mathrm{T}}$ with the corresponding minimum of the function being $f\left(\mathbf{x}_{*}\right)=-1.801303342428961 \approx-1.801$. The result validates the efficacy of the proposed method. We have tested the algorithm for many times, and the successful rate in finding the global optimizer is relatively high. In addition, when it fails to find the optimal one, the result is near optimal, i.e., it finds a local minimum.

\section{B. Example 2}

We also consider the Goldstein and Price problem [19]:

$$
\begin{array}{r}
\min _{\mathbf{x}} f(\mathbf{x})=\left[1+\left(x_{1}+x_{2}+1\right)^{2}\left(19-14 x_{1}+3 x_{1}^{2}\right.\right. \\
\left.\left.-14 x_{2}+6 x_{1} x_{2}+3 x_{2}^{2}\right)\right]\left[30+\left(2 x_{1}-3 x_{2}\right)^{2}\right. \\
\left.\left(18-32 x_{1}+12 x_{1}^{2}+48 x_{2}-36 x_{1} x_{2}+27 x_{2}^{2}\right)\right]
\end{array}
$$

The function has four local minima and the global minimizer is $\mathbf{x}_{*}=[0,-1]^{\mathrm{T}}$ with the minimum being $f_{*}=3$ in the region established by the constraints $x_{1} \geq-2$ and $x_{2} \leq 2$. By using 20 porcellio scaber and taking 40 steps of movement, with $\lambda=0.6$ and the other parameters set the same as those in the previous experiment, the best numerical experiment result of the proposed PSA for solving the Goldstein and Price problem problem (5) is visualized in Fig. 4 . Note that, the initial positions of the 20 porcellio scaber are randomly set and are guaranteed to satisfy the inequality constraints $x_{1} \geq-2$ and $x_{2} \leq 2$ so as to find the optimal solution in the region. The solution obtained in the experiment by the algorithm is $\mathbf{x}_{*}=[-0.000033275995519,-1.000060284512512]^{\mathrm{T}}$ with the corresponding function value being $f\left(\mathbf{x}_{*}\right)=$ $3.000001415798920 \approx 3$. The result further validates the efficacy of the proposed PSA. 


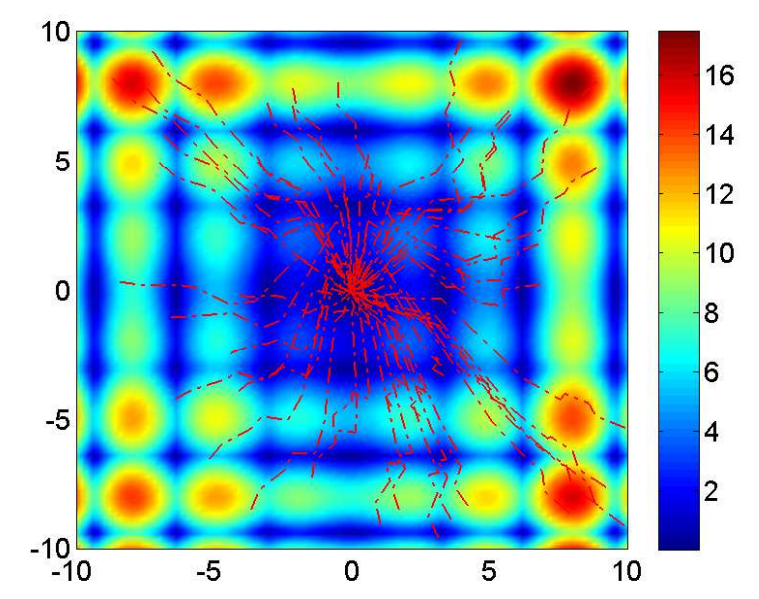

Fig. 5. Visualization of the numerical result of the proposed PSA for solving the 2-D Alpine-1-function problem 6 by using 50 porcellio scaber starting from randomly generated initial positions and by taking 100 steps of movement.

\section{Example 3}

We further consider a nondifferentiable optimization problem, i.e., the so-called 2-D Alpine-1-function problem [20]:

$$
\min _{\mathbf{x}} f(\mathbf{x})=\sum_{i=1}^{D=2}\left|x_{i} \sin \left(x_{i}\right)+0.1 x_{i}\right| .
$$

The function has many local minimizers and only one global minimizer $\mathbf{x}_{*}=[0,0]^{\mathrm{T}}$ with the the minimum being $f_{*}=0$ in the region established by the constraints $-10 \leq x_{i} \leq 10$ with $i=1,2$. By using 50 porcellio scaber and taking 100 steps of movement, with $\lambda=0.9$ and the other parameters set the same as those in the previous experiment, the best numerical experiment result of the proposed PSA for solving the 2-D Alpine-1-function problem (6) is visualized in Fig. 5. Note that, the initial positions of the 50 porcellio scaber are randomly set and are guaranteed to satisfy the inequality constraints $-10 \leq$ $x_{i} \leq 10$ so as to find the optimal solution in the region. The solution obtained in the experiment by the algorithm is $\mathbf{x}_{*}=[-0.0000066443218359,-0.000001838026950]^{\mathrm{T}} \approx$ $[0,0]^{\mathrm{T}}$ with the corresponding function value being $f\left(\mathbf{x}_{*}\right)=$ $8.478271919968875 \times 10^{-6} \approx 0$. The result further validates the efficacy of the proposed PSA for the case with a nondifferentiable objective function.

\section{CONCLusions}

In this paper, a novel bio-inspired algorithm called the PS algorithm has been proposed for solving optimization problems. Both the biology background and implementation of the proposed algorithm have been illustrated. Numerical experiment results on benchmark problems have verified the efficacy of the proposed algorithm. The extension of the algorithm to constrained optimization problems is illustrated in [21].

\section{ACKNOWLEDGMENT}

This work is supported by the Fundamental Research Funds for the Central Universities under Grant 21620346

\section{REFERENCES}

[1] M. Z. Ali, N. H. Awad, P. N. Suganthan, A. M. Shatnawi, and R. G. Reynolds, "An improved class of real-coded genetic algorithms for numerical optimization," Neurocomputing, vol. 275, pp. 155-166, 2018.

[2] Z. Lin, D. Ma, J. Meng, L. Chen, "Relative ordering learning in spiking neural network for pattern recognition," Neurocomputing, vol. 275, pp. 94-106, 2018

[3] L. Jin, S. Li,"Distributed task allocation of multiple robots: A control perspective," IEEE Transactions on Systems, Man, and Cybernetics: Systems, doi: 10.1109/TSMC.2016.2627579.

[4] C. L. P. Chen, Y. Liu, G. X. Wen, "Fuzzy neural network-based adaptive control for a class of uncertain nonlinear stochastic systems," IEEE Transactions on Cybernetics, vol. 44, no. 5, pp. 583-593, 2014.

[5] Z. Pooranian, M. Shojafar, J. H. Abawajy, A. Abraham, "An efficient meta-heuristic algorithm for grid computing," Journal of Combinatorial Optimization, vol. 30, no. 3, pp. 413-434, 2015.

[6] S. S. Gill, R. Buyya, I. Chana, M. Singh, A. Abraham, "BULLET: Particle swarm optimization based scheduling technique for provisioned cloud resources," Journal of Network and Systems Management, doi: 10.1007/s10922-017-9419-y.

[7] J. Kennedy, R. C. Eberhart, "Particle swarm optimization," Proceedings of IEEE International Conference Neural Networks, Piscataway, NJ, pp. 1942-1948, 1995

[8] M. Dorigo and L. M. Gambardella, "Ant colony system: Acooperative learning approach to the traveling salesman problem," IEEE Transactions on Evolutionary Computations, vol. 1, no. 1, pp. 53-66, 1997.

[9] X. S. Yang, S. S. S. Hosseini, and A. H. Gandomi, "Firefly algorithm for solving non-convex economic dispatch problems with valve loading effect," Applied Soft Computing, vol. 12 no. 3, pp. 1180-1186, 2012.

[10] T. T. Huan, A. J. Kulkarni, J. Kanesan, C. J. Huang, and A. Abraham, "Ideology algorithm: a socio-inspired optimization methodology," Neural Computing and Applications, vol. 28, no. 1, pp. 845-876, 2017.

[11] P. Broly, J. L. Deneubourg, and C. Devigne, "Benefits of aggregation in woodlice: a factor in the terrestrialization process," Insectes sociaux, vol. 60, no. 4, pp. 419-435, 2013.

[12] https://commons.wikimedia.org/wiki/File:Porcellio-scaber.JPG.

[13] K. E. Linsenmair, "Comparative studies on the social behaviour of the desert isopod hemilepistus reaumuri and of a porcellio species," Symposium of the Zoological Society of London, vol. 53, pp. 423-453, 1984.

[14] M. Hassalla, J. M. Tuck, "Sheltering behavior of terrestrial isopods in grasslands," Invertebrate Biology, vol. 126, no. 1, pp. 46-56, 2007.

[15] M. Pogson, "Simulation of invertebrate aggregation shows the importance of stable personality over diversity in consensus decision-making," PLos ONE vol.11, no.10, pp. 13,2011.

[16] S. Boyd and L. Vandenberghe, Convex Optimization. Cambridge, UK: Cambridge University Press, 2004.

[17] N. Andrei, "An adaptive scaled BFGS method for unconstrained optimization," Numerical Algorithms, doi: 10.1007/s11075-017-0321-1.

[18] X. S. Yang, "Firefly algorithms for multimodal optimization, International symposium on stochastic algorithms," Springer, Berlin, Heidelberg, pp. 169-178,2009.

[19] A. A. Goldstein, and J. F. Price, "Descent from local minima," Mathematics and Comptutaion, vol. 25, no. 115, pp. 569-574,1971.

[20] S. Rahnamyan, H. R. Tizhoosh, and N. M. M. Salama, "Novel population initialization method for accelerating evolutionary algorithms," Computers and Mathematics with Applications, vol.53 , no.10 pp.16051614,2007

[21] Y. Zhang, S. Li, and H. Guo, "Porcellio scaber algorithm (PSA) for solving constrained optimization problems," arXiv preprint arXiv:1710.04036 